\title{
Energy Dependence of the Scattering Operator II
}

\author{
E. B. Davies
}

Department of Mathematics, King's College, Strand, London WC2R 2LS, England

\begin{abstract}
We study the energy dependence of the scattering operator for a twobody model of electron scattering from a neutral molecule. We show that the methods of the first paper can be applied even though the dipole moment of the molecule is non-zero, and prove continuity of the scattering operator $S(E)$ as $E$ varies, in a very strong sense.
\end{abstract}

\section{Introduction}

We study the elastic scattering of an electron from a neutral molecule in the two-body approximation. That is, we study the scattering between $H=-\Delta$ and $K=H+V$ on $\mathscr{H}=L^{2}\left(\mathbb{R}^{3}\right)$, where

$$
V(x)=\int_{\mathbb{R}^{3}} \frac{1}{|x-y|} \mu(d y) .
$$

We assume that the charge distribution $\mu$ is a signed measure of finite total mass and zero net charge, that is

$$
\int \mu(d y)=0 \text {. }
$$

We also assume that the dipole moment $a$ of $\mu$, given by

$$
a=\int y \mu(d y)
$$

is non-zero, so that the potential $V$ has the asymptotic form

$$
V(x)=\frac{a \cdot x}{r^{3}}+O\left(r^{-3}\right)
$$

at infinity, where $r=|x|$. To ensure that $a$ is finite we assume $\mu$ has support within $\{x:|x| \leqq R\}$ for some $R<\infty$. Our methods could, however, easily cope with a charge distribution with exponential tails at infinity.

The potential $V$ is fairly well-behaved, and there are a variety of techniques $[1,7]$ which ensure that the wave operators between $H$ and $K$ exist and are complete. We are interested in studying the energy dependence of the scattering operator 
in the following sense. We let $\sim$ denote the unitary isomorphism of $L^{2}\left(\mathbb{R}^{3}\right)$ with $L^{2}((0, \infty), \mathscr{K})$ defined by

$$
\tilde{f}(E)(\omega)=2^{-1 / 2} E^{1 / 4} \hat{f}\left(E^{1 / 2} \omega\right),
$$

where $\mathscr{K}=L^{2}\left(S^{2}\right), E \in(0, \infty), \omega \in S^{2}$, and denotes the Fourier transform. Since the scattering operator $S$ commutes with $H$, we have

$$
(S f) \sim(E)=S(E) \tilde{f}(E)
$$

for all $f \in L^{2}\left(\mathbb{R}^{3}\right)$, where $S(E)$ are unitary operators on $\mathscr{K}$. We wish to determine the form of the operators $S(E)$ and to show that they depend continuously on $E$. Our main result is given in Theorem 10 .

We start we reviewing some recent literature. There have been some papers $[2,5,6]$ proving finiteness of the total cross-section for similar problems. However since our potential $V(x)=O\left(r^{-2}\right)$ at infinity, one does not expect the total crosssection to be finite for our problem. (This appears to be an open problem even for a pure dipole potential.) Even if the methods of $[2,5]$ could be adapted to the present problem, they would not provide information about sharp energies, but only about the average behaviour of $S(E)$ over small energy intervals.

Apart from [4], the only method we know which provides pointwise information about the scattering operators $S(E)$ is that of eigenfunction expansions. We refer to [3], which includes the class of potentials we study here, and to [1, p.421] and $[7, \mathrm{p} .107]$ which do not apply to our class of potentials unless the dipole moment vanishes. All of these approaches have the disadvantage of involving a possible exceptional null set in $(0, \infty)$, because of the use of the Fredholm alternative, and do not yield results as sharp as those of Theorem 10. It is noteworthy that we do not have any exceptional set of energies in our approach, in spite of the fact that we do not eliminate the possibility of positive point spectrum, and make no use of the nonexistence of singular continuous spectrum [7].

The main limitation of our method is that we have to assume that the charge distribution has a non-trivial symmetry group. This implies that the quadrupole moment of the potential at infinity vanishes, which is absolutely essential for the application of trace-class methods. It would be very interesting to see analogous results without this condition.

The obvious way of dealing with our problem, and one which was in our minds throughout, would be to take

$$
H^{\prime}=-\Delta+\frac{x \cdot a}{r^{3}}
$$

as the free Hamiltonian instead of $H$. Note that $H^{\prime}$ is formally of degree -2 under scaling, and the use of the scaling group is absolutely essential to our analysis, as in [4]. If the dipole moment is small enough, namely $|a|<\frac{1}{4}$, then $H^{\prime}$ can be defined as a form sum and this approach can probably be carried out. However for larger values $|a|, H^{\prime}$ is not even bounded below on $C_{c}^{\infty}\left(\mathbb{R}^{3} \backslash 0\right)$, so there is no possibility of developing a scattering theory between $K$ and any self-adjoint extension of $H^{\prime}$. Interestingly, the approach we adopt does not have any obvious discontinuities as $|a|$ increases. 


\section{Properties of the Potential}

We gather together in this section some technical calculations concerning the potential which will be used subsequently. Our main assumption is that the charge distribution $\mu$ has a non-trivial symmetry about some axis. Taking this to be the $z$-axis, we assume precisely that $\mu$ is invariant with respect to the rotation

$$
\left[\begin{array}{ccc}
\cos \theta & \sin \theta & 0 \\
-\sin \theta & \cos \theta & 0 \\
0 & 0 & 1
\end{array}\right]
$$

for some $\theta \neq 0, \pi$. Using the expansion

$$
\frac{1}{|x-y|}=\frac{1}{r}+\frac{x \cdot y}{r^{3}}+\frac{3(x \cdot y)^{2}-x^{2} y^{2}}{r^{5}}+O\left(r^{-4}\right)
$$

as $r \rightarrow \infty$, we find that

$$
V(x)=\frac{x \cdot a}{r^{3}}+\frac{\langle A x, x\rangle}{r^{5}}+O\left(r^{-4}\right)
$$

as $r \rightarrow \infty$, where the dipole moment is

$$
a=\int y \mu(d y)
$$

and the quadrupole moment is

$$
A=\int\left(\frac{3}{2}|y\rangle\langle y|-\frac{1}{2} y^{2}\right) \mu(d y) .
$$

Lemma 1. If the charge distribution has a non-trivial symmetry about some axis, and the dipole moment is non-zero, then it is possible to choose the origin on that axis so that the quadrupole moment at infinity vanishes.

Proof. Since $A$ is a real symmetric matrix of zero trace, and since $a$ and $A$ are both invariant with respect to $U$, they must have the forms

$$
a=\left[\begin{array}{c}
0 \\
0 \\
|a|
\end{array}\right], \quad A=\left[\begin{array}{ccc}
\beta & 0 & 0 \\
0 & \beta & 0 \\
0 & 0 & -2 \beta
\end{array}\right],
$$

so that

$$
A=\beta\left(1-\frac{3}{a^{2}}|a\rangle\langle a|\right) .
$$

Now changing the origin by

$$
y^{\prime}=y+\lambda a
$$

the dipole moment is unaltered, while the quadrupole moment becomes

$$
\begin{aligned}
A & =\int\left(\frac{3}{2}|y+\lambda a\rangle\langle y+\lambda a|-\frac{1}{2}(y+\lambda a)^{2}\right) \mu(d y) \\
& =A+3 \lambda|a\rangle\langle a|-\lambda a^{2},
\end{aligned}
$$

which vanishes if $\lambda=\beta / a^{2}$. 
For the rest of this paper we assume that the origin can be chosen so that the quadrupole moment of the potential at infinity vanishes (this actually uniquely determines the origin). The symmetry of the charge distribution will not be used again.

Lemma 2. The potential $V(x)$ is $L^{2}$ within $\{|x| \leqq 2 R\}$. It is also $C^{\infty}$ within $\{|x|>R\}$ and has asymptotic form

$$
V(x)=\frac{a \cdot x}{r^{3}}+O\left(r^{-4}\right)
$$

at infinity. In particular $V \in L^{2}\left(\mathbb{R}^{3}\right)$.

Of central importance in this paper will be the scaling group $R_{s}$ defined on $L^{2}\left(\mathbb{R}^{3}\right)$ by

$$
\left(R_{s} f\right)(x)=e^{-3 s / 4} f\left(e^{-s / 2} x\right),
$$

and in the representation $L^{2}((0, \infty), \mathscr{K})$ by

$$
\left(R_{s} f\right)^{\tilde{\sim}}(E)=e^{s / 2} \tilde{f}\left(e^{s} E\right) .
$$

It is easy to verify that the free Hamiltonian $H$ has the scaling property

$$
H=e^{-s} R_{s} H R_{-s} .
$$

We now define the potential $V_{s}$ on $\mathbb{R}^{3}$ by

$$
V_{s}=e^{-s} R_{s} V R_{-s}
$$

or equivalently

$$
V_{s}(x)=e^{-s} V\left(e^{-s / 2} x\right) .
$$

Lemma 3. The potential $\left(V_{s+t}-V_{s}\right)$ lies in $L^{1}\left(\mathbb{R}^{3}\right)$ for all $s, t \in \mathbb{R}$. Moreover

$$
\lim _{t \rightarrow 0} t^{-1}\left(V_{s+t}-V_{s}\right)=W_{s}
$$

exists as an $L^{1}$ norm limit for all $s \in \mathbb{R}$. The potential $W_{s} \in L^{1}\left(\mathbb{R}^{3}\right)$ satisfies

$$
W_{s}=e^{-s} R_{s} W_{0} R_{-s}
$$

and depends continuously upon $s$ in $L^{1}$ norm.

Proof. If we put

$$
X(x)=\frac{1}{|x-y|}
$$

and

$$
X_{s}(x)=e^{-s} R_{s} X R_{-s}(x)=\frac{e^{-s / 2}}{\left|x-e^{s / 2} y\right|},
$$


then formally at least we have

$$
\frac{\partial}{\partial s} X_{s}(x)=\frac{1}{2} \frac{\left(x-e^{s / 2} y\right) \cdot y}{\left|x-e^{s / 2} y\right|^{3}}-\frac{1}{2\left|x-e^{s / 2} y\right|} .
$$

Therefore the appropriate definition of $W_{s}$ is

$$
W_{s}(x)=\frac{1}{2} e^{-s / 2}\left\{\left\{\frac{\left(x-e^{s / 2} y\right) \cdot e^{s / 2} y}{\left|x-e^{s / 2} y\right|^{3}}-\frac{1}{\left|x-e^{s / 2} y\right|}\right\} \mu(d y),\right.
$$

which is the potential due to a mixed distribution of charges and dipoles. The remainder of the proof consists of a rigorous verification that this choice of $W_{s}$ has all the stated properties.

Since each term of the integrand is $L^{1}$ within $\{|x| \leqq 2 R\}$ so is $W_{s}$. Moreover $W_{s}$ is clearly $C^{\infty}$ in $\{|x|>R\}$ and tends to zero at infinity. Moreover as $|x| \rightarrow \infty$

$$
\begin{aligned}
& \frac{(x-u) \cdot u}{|x-u|^{3}}-\frac{1}{|x-u|} \\
& =-\frac{1}{|x|}+\frac{3(x \cdot u)^{2}}{2|x|^{5}}-\frac{|u|^{2}}{2|x|^{3}}+O\left(|x|^{-4}\right) .
\end{aligned}
$$

Therefore

$$
\begin{aligned}
W_{s}(x)= & \frac{1}{2} e^{-s / 2} \int\left\{-\frac{1}{|x|}+\frac{3\left(x \cdot e^{s / 2} y\right)^{2}}{2|x|^{5}}\right. \\
& \left.-\frac{\left|e^{s / 2} y\right|^{2}}{2|x|^{3}}+O\left(|x|^{-4}\right)\right\} \mu(d y) \\
= & -\frac{e^{-s / 2}}{2|x|} \int \mu(d y)+e^{s} \frac{\langle A x, x\rangle}{|x|^{5}}+O\left(|x|^{-4}\right)
\end{aligned}
$$

as $|x| \rightarrow \infty$. But the total charge and the quadrupole moment of $\mu$ vanish by hypothesis so

$$
W_{x}(x)=O\left(|x|^{-4}\right)
$$

as $|x| \rightarrow \infty$, and $W_{s} \in L^{1}\left(\mathbb{R}^{3}\right)$. The identity (2) is elementary and implies that $W_{s}$ depend continuously on $s$ in $L^{1}$ norm. The rigorous proof of (1) is now obtained by verifying that

$$
V_{s}-V_{0}=\int_{0}^{s} W_{t} d t
$$

both pointwise and in $L^{1}$ norm.

\section{Some Operator Theory}

We combine the above calculations with a modification of the theory of [4], with which this section must be read. We write $\mathscr{T}(\mathscr{K})$ for the space of trace-class operators on $\mathscr{K}$ with the trace norm \|\|$_{1}$, and define $\mathscr{B}$ as in [4] to be the Banach 
space of all integrable $\mathscr{T}(\mathscr{K})$-valued functions on $(0, \infty)$ with the norm

$$
\|f\|=\int_{0}^{\infty}\|f(E)\|_{1} d E
$$

We also let $M(H)$ be the set of $\phi$ in $L^{2}((0, \infty), \mathscr{K})$ which are essentially bounded, and define the contraction $\&$ from $\mathscr{T}(\mathscr{H})$ to $\mathscr{B}$ by

$$
\int_{0}^{\infty}\left\langle X^{\mathrm{\natural}}(E) \phi(E), \psi(E)\right\rangle d E=\frac{1}{2 \pi} \int_{-\infty}^{\infty}\left\langle e^{-i H s} X e^{i H s} \phi, \psi\right\rangle d s
$$

for all $\phi, \psi \in M(H)$ and $X \in \mathscr{T}(\mathscr{H})$, so that

$$
\int_{0}^{\infty} \operatorname{tr}\left[X^{\mathrm{\natural}}(E)\right] d E=\operatorname{tr}[X]
$$

Unfortunately the factor $\frac{1}{2 \pi}$ on the right hand side of (3) was omitted in Theorem 2 of [4], which causes various minor errors later in that paper. In particular the constants on the right hand side of various estimates in [4], for example (8), (9), (24), (37), (38), (40) must be increased by factors of $2 \pi$. We thank G. Coombes for pointing out this error to us.

If $S_{s}$ is the scattering operator for

$$
K_{s}=H+V_{s}=e^{-s} R_{s}(H+V) R_{-s},
$$

then it follows as in [4, Lemma 7] that

$$
S_{s}(E)=S\left(e^{s} E\right)
$$

for all $s \in \mathbb{R}$ and a.e. $E \in \mathbb{R}^{+}$.

Lemma 4. If $\phi, \psi \in M(H)$ and $\alpha>0$ is large enough then

$$
\begin{gathered}
\left\langle(H+\alpha)^{-1}\left(S_{s}-S_{0}\right)(H+\alpha)^{-1} \phi, \psi\right\rangle \\
=\int_{-\infty}^{\infty}\left\langle e^{-i H s} X e^{i H s} \phi, \psi\right\rangle d s,
\end{gathered}
$$

where $X \in \mathscr{T}(\mathscr{H})$ is defined by

$$
X=-i \Omega_{0}^{+*}\left(K_{0}+\alpha\right)^{-1}\left(V_{s}-V_{0}\right)\left(K_{s}+\alpha\right)^{-1} \Omega_{s}^{-} .
$$

Hence $S_{s}(E)-S_{0}(E)$ is of trace class for a.e. $E$, and

$$
\int_{0}^{\infty} \frac{\left\|S_{s}(E)-S_{0}(E)\right\|_{1}}{(E+\alpha)^{2}} d E \leqq 2 \pi\left\|\left(K_{0}+\alpha\right)^{-1}\left(V_{s}-V_{0}\right)\left(\mathrm{K}_{s}+\alpha\right)^{-1}\right\|_{1} .
$$

Proof. This follows the proof of Theorem 3 of [4]. The assumption there that $V_{0}, V_{s}$ were in $L^{1}\left(\mathbb{R}^{3}\right)$ (as well as their difference) was only used to ensure the existence and completeness of the wave operators. In our context, however, this may be proved by a variety of other techniques $[1,7]$. We use the Kato-Birman theory only to study 
the scattering between $K_{0}$ and $K_{s}$. The identity

$$
\Omega_{s}^{ \pm} M(H) \subseteq M\left(K_{s}\right)
$$

does not depend upon the Kato-Birman theory but only on the definitions and the existence of the wave operators. The proof that $X$ is trace class depends upon writing $X=A B C D$ where

$$
\begin{aligned}
& A=-i \Omega_{0}^{+*}\left(K_{0}+\alpha\right)^{-1}(H+\alpha), \\
& B=(H+\alpha)^{-1}\left|V_{s}-V_{0}\right|^{1 / 2}, \\
& C=\left(V_{s}-V_{0}\right)\left|V_{s}-V_{0}\right|^{-1 / 2}(H+\alpha)^{-1}, \\
& D=(H+\alpha)\left(K_{s}+\alpha\right)^{-1} \Omega_{s}^{-} .
\end{aligned}
$$

The operators $A$ and $D$ are bounded since $H, K_{0}$ and $K_{s}$ all have the same domain, while $B$ and $C$ are Hilbert-Schmidt because $\left|V_{s}-V_{0}\right|^{1 / 2}$ lies in $L^{2}\left(\mathbb{R}^{3}\right)$.

Lemma 5. If $B_{s} \in \mathscr{B}$ is defined by

$$
B_{s}(E)=\frac{S\left(e^{s} E\right)-S(E)}{s(E+\alpha)^{2}},
$$

then $B_{s}$ is norm convergent as $s \rightarrow 0$.

Proof. We see from Lemma 4 that

$$
B_{s}=2 \pi Y_{s}^{\natural},
$$

where $Y_{s} \in \mathscr{T}(\mathscr{H})$ is defined by

$$
Y_{s}=A B_{s} C_{s} \Omega_{s}^{-}
$$

and

$$
\begin{aligned}
A & =-i \Omega_{0}^{+*}\left(K_{0}+\alpha\right)^{-1}(H+\alpha), \\
B_{s} & =(H+\alpha)^{-1} s^{-1}\left(V_{s}-V_{0}\right)(H+\alpha)^{-1}, \\
C_{s} & =(H+\alpha)\left(K_{s}+\alpha\right)^{-1} .
\end{aligned}
$$

Now by Lemma $3, B_{s}$ converges in trace norm to

$$
B=(H+\alpha) W_{0}(H+\alpha)^{-1} \in \mathscr{T}(\mathscr{H})
$$

as $s \rightarrow 0$. Also

$$
C_{s}^{-1}=\left(K_{s}+\alpha\right)(H+\alpha)^{-1}=1+V_{s}(H+\alpha)^{-1}
$$

converges in operator norm to

$$
1+V(H+\alpha)^{-1}=\left(K_{0}+\alpha\right)(H+\alpha)^{-1}
$$

as $s \rightarrow 0$, so for large enough $\alpha>0, C_{s}$ converges in operator norm to

$$
C=(H+\alpha)\left(K_{0}+\alpha\right)^{-1}
$$

as $s \rightarrow 0$. 
Finally

$$
\begin{aligned}
\Omega_{s}^{-1} & =\lim _{u \rightarrow \infty} e^{-i\left(H+V_{s}\right) u} e^{i H u} \\
& =\lim _{u \rightarrow \infty} R_{s} e^{-i(H+V) u} e^{i H u} R_{-s} \\
& =R_{s} \Omega_{0}^{-} R_{-s} .
\end{aligned}
$$

Therefore $\Omega_{s}^{-*}$ converges strongly to $\Omega_{0}^{-*}$ as $s \rightarrow 0$. Combining these results we deduce that $Y_{t}$ converges in trace norm to $A B C \Omega_{0}^{-}$as $s \rightarrow 0$.

\section{The Main Theorems}

If we let $s \rightarrow 0$ formally in (4) we find that there exists $T \in \mathscr{B}=L^{1}((0, \infty), \mathscr{T}(\mathscr{K}))$ such that

$$
\frac{E S^{\prime}(E)}{(E+\alpha)^{2}}=T(E) \text {. }
$$

This enables us to deduce that for all $0<E<F<\infty$

$$
S(F)-S(E)=\int_{E}^{F} \frac{(x+\alpha)^{2} T(x)}{x} d x,
$$

so that although $S(E)-1$ need not be trace class for any $E \in \mathbb{R}^{+}, S(F)-S(E)$ is trace class for all $E, F \in \mathbb{R}^{+}$. Most of this section is taken up by a rigorous proof of (5).

Lemma 6. If $T \in \mathscr{B}$ and the continuous $\mathscr{T}(\mathscr{K})$-valued function on $(0, \infty)$ is defined by

$$
R(E)=\int_{\alpha}^{E} \frac{(x+\alpha)^{2}}{x} T(x) d x
$$

then

$$
\int_{0}^{\infty} \frac{\left\|R\left(e^{s} E\right)-R(E)\right\|_{1}}{(E+\alpha)^{2}} d E \leqq\left(e^{|s|}-1\right) \int_{0}^{\infty}\|T(x)\|_{1} d x .
$$

Proof. Since

$$
R\left(e^{s} E\right)-R(E)=\int_{E}^{e^{s}} \frac{(x+\alpha)^{2}}{x} T(x) d x,
$$

the left hand side of (6) is dominated for $s>0$ by

$$
\begin{aligned}
& \int_{0<E<x<e^{s} E<\infty} \frac{(x+\alpha)^{2}}{x(E+\alpha)^{2}}\|T(x)\|_{1} d x d E \\
= & \iint_{0<e^{-} s_{x}<E<x<\infty} \frac{(x+\alpha)^{2}}{x(E+\alpha)^{2}}\|T(x)\|_{1} d x d E
\end{aligned}
$$




$$
\begin{aligned}
& =\int_{0}^{\infty}\left(1-e^{-s}\right) \frac{x+\alpha}{e^{-s} x+\alpha}\|T(x)\|_{1} d x \\
& \leqq\left(e^{s}-1\right) \int_{0}^{\infty}\|T(x)\|_{1} d x .
\end{aligned}
$$

The proof for $s<0$ is similar.

Lemma 7. In the circumstances of Lemma 6 one has

$$
\lim _{s \rightarrow 0} s^{-1}\left\{R\left(e^{s} E\right)-R(E)\right\}=T(E) .
$$

provided (7) is interpreted as an $L^{1}$ norm limit.

Proof. It follows from Lemma 6 that

$$
\int_{0}^{\infty}|s|^{-1}\left\|R\left(e^{s} E\right)-R(E)\right\|_{1} d E \leqq(e-1) \int_{0}^{\infty}\|T(E)\|_{1} d E,
$$

provided $|s| \leqq 1$ and $T \in \mathscr{B}$. By approximation arguments it is therefore sufficient to prove (7) for a set of $T$ which generate $\mathscr{B}$. Such a set is given by

$$
T(x)=\frac{x}{(x+\alpha)^{2}} \lambda e^{-\lambda x} b,
$$

where $0<\lambda<\infty$ and $b \in \mathscr{T}(\mathscr{K})$. For this choice of $T$

$$
R(E)=\left(e^{-\lambda \alpha}-e^{-\lambda E}\right) b
$$

so

$$
\frac{R\left(e^{s} E\right)-R(E)}{s(E+\alpha)^{2}}=\frac{e^{-\lambda E} b}{(E+\alpha)^{2}} S^{-1}\left\{1-e^{-\lambda E\left(e^{s}-1\right)}\right\} .
$$

This converges pointwise, and also in $L^{1}$ norm by the dominated convergence theorem, to $T$.

We now take $T$ to be the norm limit as $s \rightarrow 0$ of $B_{s}$, defined by (4) and we define $A(E)$ by

$$
A(E)=S(E)-R(E)
$$

so that, formally at least, A has zero derivative.

Lemma 8. The operator $A(E)$ is bounded for a.e. $E$ and

$$
\int_{0}^{\infty} \frac{E\left|\left\langle A\left(e^{s} E\right) \phi, \psi\right\rangle\right|}{(E+\alpha)^{4}} d E<\infty
$$

for all $s \in \mathbb{R}$ and $\phi, \psi \in \mathscr{K}$. Moreover

$$
\lim _{s \rightarrow 0} \int_{0}^{\infty} \frac{E\left|\left\langle A\left(e^{s} E\right) \phi, \psi\right\rangle-\langle A(E) \phi \cdot \psi\rangle\right|}{s(E+\alpha)^{4}} d E=0 .
$$


Proof. Since $\frac{(x+\alpha)^{2}}{x}$ takes its minimum value at $x=\alpha$ we see that

$$
\begin{aligned}
\|R(E)\|_{1} & \leqq \frac{(E+\alpha)^{2}}{E} \int_{\alpha}^{E}\|T(x)\|_{1} d x \\
& \leqq \frac{(E+\alpha)^{2}}{E} \int_{0}^{\infty}\|T(x)\|_{1} d x .
\end{aligned}
$$

Therefore

$$
\begin{aligned}
\|A(E)\| & \leqq 1+\|R(E)\| \\
& \leqq 1+\frac{(E+\alpha)^{2}}{E}
\end{aligned}
$$

for all $0<E<\infty$, which implies (8). Now

$$
\frac{A\left(e^{s} E\right)-A(E)}{s(E+\alpha)^{2}}=\frac{S\left(e^{s} E\right)-S(E)}{s(E+\alpha)^{2}}-\frac{R\left(e^{s} E\right)-R(E)}{s(E+\alpha)^{2}}
$$

lies in $\mathscr{B}$ for all $s \in \mathbb{R}$ and converges in $\mathscr{B}$-norm to zero as $s \rightarrow 0$ by Lemmas 5 and 7. Therefore

$$
\begin{gathered}
\int_{0}^{\infty} \frac{E\left|\left\langle A\left(e^{s} E\right) \phi, \psi\right\rangle-\langle A(E) \phi, \psi\rangle\right|}{s(E+\alpha)^{4}} d E \\
\leqq \frac{\|\phi\|\|\psi\|}{\alpha} \int_{0}^{\infty} \frac{\left\|A\left(e^{s} E\right)-A(E)\right\|_{1}}{s(E+\alpha)^{2}} d E
\end{gathered}
$$

converges to zero as $s \rightarrow 0$.

Theorem 9. There exists a fixed bounded operator $A$ on $\mathscr{K}$ and a function $T \in L^{1}((0, \infty), \mathscr{T}(\mathscr{K}))$ such that

$$
S(E)=A+\int_{\alpha}^{E} \frac{(x+\alpha)^{2}}{x} T(x) d x
$$

for a.e. $E$ with $0<E<\infty$. In particular $S(E)$ is (equal a.e. to) a continuous function of $E$ with

$$
\lim _{F \rightarrow E}\|S(F)-S(E)\|_{1}=0
$$

for all $0<E<\infty$.

Proof. We finally show that $A(E)$ is equal a.e. to a fixed bounded operator. Let $\mathscr{C}$ be the Banach space of measurable complex-valued functions on $(0, \infty)$ with the norm

$$
\|f\|=\int_{0}^{\infty} \frac{x|f(x)|}{(x+\alpha)^{4}} d x
$$

and let $U_{s}$ be the strongly continuous one-parameter group on $\mathscr{C}$ defined by

$$
U_{s} f(x)=f\left(e^{s} x\right) .
$$


We have shown that

$$
f(x)=\langle A(x) \phi, \psi\rangle
$$

lies in the domain of the generator $Z$ of $U_{s}$ with $Z f=0$. Therefore $U_{s} f=f$ for all $s \in \mathbb{R}$, so

$$
f=\int_{0}^{1} U_{s} f d s
$$

or

$$
f(x)=\int_{0}^{1} f\left(e^{s} x\right) d s
$$

for a.e. $x$. But the right hand side of (9) is a continuous function of $x$, so $f$ is (equal a.e. to) a continuous function. Using the identity $U_{s} f=f$ again we see that $f$ is constant. Thus $\langle A(x) \phi, \psi\rangle$ is equal a.e. to a constant for all $\phi, \psi \in \mathscr{K}$, and this implies that there exists a bounded operator $A$ such that $A(x)=A$ a.e.

The above theorem does not identify the operator $A$. This is done in the proof of the following theorem.

Theorem 10. There exists a bounded operator $B$, depending on the dipole moment of the charge distribution alone, such that $(S(E)-B)$ is a trace class operator for all $E>0$, and such that $(S(E)-B)$ depends continuously upon $E$ in trace norm.

Proof. We repeat the calculations of this paper for the Hamiltonian $K^{\prime}=H+V^{\prime}$. where

$$
V^{\prime}(x)=\frac{x \cdot a}{\left(x^{2}+a^{2}\right)^{3 / 2}}
$$

and $a$ is the dipole moment of the original charge distribution $\mu$. We discover that the scattering operator $S^{\prime}$ has the form

$$
S^{\prime}(E)=B+\int_{\alpha}^{E} \frac{(x+\alpha)^{2}}{x} T^{\prime}(x) d x,
$$

where $T^{\prime} \in \mathscr{B}$. Now by a modification of Lemma 4 we see that

$$
\int_{0}^{\infty} \frac{\|S(E)-S(E)\|_{1}}{(E+\alpha)^{2}} d E<\infty,
$$

and this implies that $(A-B)$ is of trace class.

Note 1 . The operator $B$ is not uniquely determined by Theorem 10 , but only up to the addition of a trace class operator. However if the dipole moment is small enough, we can find a canonical representative for $B$. Namely if $|a|<\frac{1}{4}$, then we can take the comparison Hamiltonian to be

$$
K^{\prime}=H+\frac{a \cdot x}{r^{3}}
$$


as explained in Sect. 1. Now $H$ and $K^{\prime}$ are both homogeneous of degree -2 under scaling, so $S(E)=B$ is independent of $E$. We conjecture that if $B$ were evaluated by this method for $|a|<\frac{1}{4}$, then it would turn out to be an analytic function of $|a|$ for all 0 $<|a|<\infty$, and that the operator so obtained would be a suitable choice for $B$ even when $|a| \geqq \frac{1}{4}$.

Note 2. It is obvious that the theory we have developed could be used to obtain explicit pointwise bounds on

$$
\|S(E)-S(E)\|_{1}
$$

as $E$ and $F$ vary, with some further effort. Although we know of no other method of obtaining such bounds, we do not believe that those obtained by this method will be optimal. For the high energy behaviour of $(S(E)-B)$ probably depends upon the nature of the local singularities of $W_{s}$. We have only used the fact that these potentials lie in $L^{1}(\mathbb{R})^{3}$, which allows considerable worse local singularities than $W_{s}$ actually possesses.

\section{References}

1. Amrein, W. O., Jauch, J. M., Sinha, K. B.: Scattering theory in quantum mechanics. New York, W. A. Benjamin, Inc. 1977

2. Amrein, W. O., Pearson, D. B.: A time-dependent approach to the total scattering cross-section. J. Phys. A12 1469-1492 (1979)

3. Amrein, W. O., Pearson, D. B.: Commutators of Hilbert-Schmidt type and the scattering crosssection. Ann. Inst. H. Poincaré 30, 89-107 (1979)

4. Davies, E. B.: Energy dependence of the scattering operator. Adv. Appl. Math. 1, 300-323 (1980)

5. Enss, V., Simon, B.: Finite total cross-sections in nonrelativistic quantum mechanics. Commun. Math. Phys. 76, 177-209 (1980)

6. Martin, A.: Boundedness of total cross-sections in potential scattering. Commun. Math. Phys. 69, 8998 (1979)

7. Reed, M., Simon, B.: Methods of modern mathematical physics, Vol. 3. New York: Academic Press 1979 